\title{
La Reforma de la Arquitectura FINANCIERA INTERNACIONAL
}

\author{
Dra. Beatriz Herrera Garcia
}

Vicerrectora Administrativa

\begin{abstract}
RESUMEN
La aplicación, la evaluación y el perfeccionamiento de las políticas, significa que la asistencia técnica centrada en las necesidades particulares de cada país se ha convertido en un requisito clave para la eficacia de la labor de reforma de la arquitectura financiera internacional. La asistencia técnica y el apoyo resultan decisivos para asegurar que ningún país quede al margen de los esfuerzos encaminados a aumentar la capacidad de recuperación financiera en todo el mundo. Sin embargo, los episodios de crisis registrados en Argentina y Turquía constituyen una clara advertencia de que aún queda mucho por hacer en materia de reforma de la arquitectura financiera internacional. Por otra parte, los responsables de la adopción de políticas tienen que estar dispuestos a hacer frente a posibles perturbaciones del sistema financiero y de la economía real, causada por desequilibrios creados durante la última y larga fase de expansión económica.
\end{abstract}

Palabras clave: Reforma, sistema financiero, bancos, mercado de capitales, sector privado.

\section{INTRODUCCIÓN}

La crisis financiera asiática de mediados de 1997, su internacionalización y sus precedentes en los años ochenta (crisis de la deuda) y noventa (Efecto Tequila, México 1994), han puesto de manifiesto en forma dramática que el actual sistema financiero internacional es incapaz de proteger a la economía mundial de las intensas y frecuentes crisis financieras. Estas crisis han sido un reflejo de la tendencia de los mercados financieros a experimentar ciclos económicos oscilantes y agudos que transcurren por fases de reanimación, auge, declinación y crisis o depresión ${ }^{(1)}$.

Lo nuevo de las crisis financieras de los noventa ha sido su efecto contagio internacional, creando pánico en los mercados sin distinguir entre economías sólidas y débiles. El efecto contagio se da porque en los mercados financieros internacionales se concentran participantes que aplican similares criterios de decisión a todos los países; por lo que las economías en desarrollo han sido muy vulnerables a la volatilidad financiera y al contagio. Han sido propensas a períodos de acelerada expansión y diversificación de los flujos financieros, que suelen ser seguidas de reversiones abruptas. La liberalización prematura y apresurada de la cuenta de capitales, la fragilidad de la estructura financiera internacional doméstica, y la debilidad de la regulación y la supervisión financieras, han acentuado ese patrón.

Las crisis de los años noventa han puesto en evidencia que las instituciones financieras internacionales no son las adecuadas para enfrentar la globalización financiera ${ }^{(2)}$. La deficiencia sistémica y la consiguiente amenaza de nuevas crisis futuras han puesto de manifiesto la necesidad de reformar el sistema financiero internacional. 


\section{BASES PARA GESTIONAR LAS CRISIS FINANCIERAS}

La reforma del sistema financiero internacional debe abarcar el manejo de la liquidez internacional, la consistencia global de las políticas macroeconómicas y las regulaciones financieras, porque son factores estrechamente interrelacionados.

El entorno económico internacional es también un factor decisivo en la propagación de la crisis y puede ser determinante en el proceso de recuperación. Los riesgos de crisis, propagación y recuperación lenta son menores en una economía mundial que goza de un crecimiento considerable y sostenido, una baja inflación y cierta estabilidad en los mercados monetarios internacionales. Este enfoque es más probable cuando existe coordinación entre políticas macroeconómicas, en el comercio y las corrientes financieras mundiales. En ese sentido hay avances por el lado del Grupo de los Siete ${ }^{(3)}$. Por su parte, el Fondo Monetario Internacional (FMI), con sus políticas de doble condicionalidad hacia los países en desarrollo, supervisa las políticas macroeconómicas de cada uno de los miembros del Grupo, sin embargo, apremia menos a que se acate las recomendaciones normativas del Fondo, a diferencia del trato y las exigencias hacia los países en desarrollo.

En relación al fortalecimiento del sector financiero, las autoridades nacionales e internacionales han adoptado diversas medidas desde que se produjeron las crisis, para mejorar la disponibilidad de dicha información y su comparación a nivel internacional. Los gobiernos suelen otorgar a los bancos una "red de seguridad"(4), que va más allá de la protección que se brinda a otros sectores del sistema financiero, sin embargo, la red de seguridad conlleva un riesgo moral, es decir, invita a que los bancos asuman una conducta riesgosa porque las probabilidades de quiebra inspiran a su dirección menos temores que albergarían de otro modo.

En períodos de crisis, el pánico bancario representa en la economía un caso de comportamiento de "manada"; y una de las razones por la que se manifiesta el instinto de manada entre los ahorristas y se difunde el contagio de los bancos débiles a los fuertes, es que el público no puede evaluar fácilmente el grado de riesgo que representa el balance de un banco, esa incertidumbre deja a los bancos expuestos al pánico. Asimismo, es fundamental la posibilidad de que se genere un pánico bancario a través de dos características claves de los sistemas bancarios: la intermediación y el endeudamiento relativo al capital ${ }^{(5)}$. Es decir, el riesgo de liquidez de los bancos se manifiesta por su nivel de endeudamiento relativo al capital y viceversa. Por lo tanto, los bancos necesitan tener una red de seguridad para proteger a la economía del riesgo propio del sistema, que un pánico bancario podría plantear a toda la economía. Si un banco no cumpliera sus obligaciones con sus ahorristas, el contagio consiguiente podría llevar al derrumbe del sistema bancario y de pagos y arrasar consigo al resto de la economía.

Por lo indicado, una red de seguridad moderna debe contener tres instrumentos: prestamista de última instancia, la gestión de la crisis y un sistema de seguro de depósitos. Como instrumento de prestamista de última instancia, el banco central debe anunciar, que en caso se produzca una crisis estará dispuesto a conceder a los bancos préstamos voluminosos mediante una garantía adecuada, pero con un tipo de interés punitivo - para desalentar el abuso de la línea de crédito.

A fines de 1998, frente a las reacciones del mercado financiero ante la devaluación del rublo y la suspensión del pago del servicio de la deuda rusa, la Reserva Federal de 
Estados Unidos inició operaciones en el mercado libre -adquirió bonos del Estado-y de ese modo inyectó liquidez al sistema bancario. Por otro lado, el Banco de Reserva de Nueva York procuró enfrentar la probable crisis de liquidez del Fondo de Gestión de Capitales a Largo Plazo (LTCM), un fondo de salvaguardia que había tomado importantes préstamos en varios bancos grandes y había perdido grandes sumas de dinero; mediante la coordinación de una voluminosa transferencia de fondos vía créditos de los bancos privados, con lo que el Fondo de Gestión comenzó a desenredar su compleja situación financiera y su elevado endeudamiento en relación a su capital. Es decir, se gestionó la crisis a través de medidas que involucraban directamente a la institución en crisis.

El tercer instrumento trata de un sistema de seguro de depósitos que protege a los pequeños ahorristas en caso de quiebra del banco. Esa disminución del riesgo constituye un servicio social y económicamente valioso que resolvía lo que se consideraba un grave problema en la época, en que se creó por primera vez el seguro de depósitos -durante la depresión de los años treinta.

De acuerdo a las experiencias acumuladas, desde entonces, varios países han tomado iniciativas para cerrar o consolidar los bancos quebrados; abrir el sector bancario a una mayor participación extranjera, fortalecer la reglamentación bancaria y aumentar la capacidad de supervisión de los organismos responsables de hacerla cumplir.

\section{TRANSPARENCIA DE LOS FLUJOS DE INFORMACIÓN Y FORTALECIMIENTO DE LA SUPERVISIÓN Y LA REGULACIÓN FINANCIERAS}

Los especialistas sostienen que si se aumenta la cantidad y mejora la calidad de la información, mejoraría la capacidad de adopción de decisiones de los inversionistas nacionales e internacionales, y se dispondría de una fuente de alerta anticipada de crisis. También, la confianza de los mercados financieros internacionales y la alerta anticipada pueden mejorarse con evaluaciones convincentes de la situación económica general de cada uno de los países. Con arreglo a una nueva política que se inició en 1997, el Fondo adoptó un criterio más sistemático para publicar las notas informativas públicas, que son resúmenes de las deliberaciones del Directorio Ejecutivo -que suelen enviarse al gobierno del país evaluado por el FMI, estos memorandos tenían carácter confidencial. Tal aumento de transparencia ha sido generalmente bien recibido.

Además, también están los códigos de buenas prácticas que pretenden servir de directivas amplias, que tendrán que adaptarse a las circunstancias de cada país. Entre éstas encontramos:

- Las Normas Especiales de Difusión de Datos;

- El Código de Buenas Prácticas de Transparencia Fiscal (1998); y

- El Código de Buenas Prácticas de Transparencia en las Políticas Monetarias y Fiscales (1999).

El Acuerdo de Basilea, producto de las deliberaciones del Grupo de los Diez (1988) para establecer normas comunes relativas a la suficiencia de capital social para sus bancos comerciales que operan en el extranjero ${ }^{(6)}$, se convirtió en el parámetro regulatorio de la década de los noventa para el sector bancario. Sin embargo, los reguladores tuvieron problemas para seguir el ritmo de una industria financiera cada vez más mundializada y en rápida transformación. 
La crisis financiera de 1997 obligó a la actualización y ampliación de las diversas normas regulatorias internacionales, por lo que el Comité de Basilea de Supervisión Bancaria publicó en enero de 1998 un informe en el que se analizaban las relaciones de los bancos con instituciones que tuvieran un alto coeficiente de endeudamiento -sobre todo fondos especulativos de cobertura-y se ofrecía orientación sobre prácticas seguras para transacciones de ese tipo. En julio de 1999, el Comité publicó cuatro informes en los que se ofrecía orientación a los bancos y supervisores bancarios sobre diversos aspectos del riesgo crediticio en las actividades bancarias.

Además, el Foro de Estabilidad Financiera creado en febrero de 1999, con el fin de integrar y reforzar las diversas actividades regulatorias y actividades conexas, creó tres grupos de trabajo que presentaron informes en setiembre de 1999: a) sobre las medidas para reducir el potencial desestabilizador de las instituciones con alto coeficiente de endeudamiento; b) sobre las medidas para reducir la inestabilidad de las corrientes de capital y los riesgos para los sistemas financieros con excesivo endeudamiento a corto plazo; c) sobre las consecuencias para la estabilidad financiera mundial de los centros financieros transnacionales y, d) sobre el cumplimiento de las normas prudenciales internacionales y los acuerdos para el intercambio transfronterizo de información.

Por otro lado, se sostiene que los países empezaron a ser más vulnerables a la inestabilidad y al contagio, cuando en la década de los años noventa relajaron los controles impuestos a las corrientes internacionales de capital, incluidas las corrientes especulativas de crédito a corto plazo. Incluso antes de la última serie de crisis financieras, las recomendaciones para acelerar la liberalización de las corrientes de capital en economías en desarro- llo fueron controvertidas. A la luz de la experiencia más reciente, los defensores de la liberalización han matizado más su posición.

Las crisis financieras y su propagación también se pueden evitar reforzando los recursos financieros externos de que disponen los países para adoptar medidas preventivas. Además de acumular cantidades considerables de reservas oficiales en el banco central, algunos gobiernos han negociado líneas de crédito contingente ${ }^{(7)}$ con acreedores privados extranjeros.

En 1998, se efectuaron retiros de dos mecanismos de crédito específicos del FMI: Los Acuerdos Generales, se utilizaron para apoyar el programa de ajuste de Rusia, aplicado en julio, y los Nuevos Acuerdos, se utilizaron en diciembre para ayudar a financiar el programa de ajuste del Brasil. Los fondos movilizados para contrarrestar las crisis financieras exigieron la participación del Banco Mundial, además del Fondo. La gran mayoría de los fondos proporcionados no benefició a las poblaciones de los países en que se produjeron las crisis, sino que financió en gran parte el endeudamiento con los acreedores privados extranjeros e incluso facilitó la fuga de capitales. El apoyo a las medidas oficiales de rescate financiero se vio debilitado por la idea generalizada de que se estaba rescatando a los acreedores privados, tanto extranjeros como nacionales, con fondos públicos. Por lo que la comunidad financiera internacional optó por otros mecanismos, en los cuales los acreedores privados extranjeros tendrían más participación en la solución de emergencias, por ejemplo, renovando los créditos vencidos.

Por otro lado, la concesión de préstamos a países con atrasos no soluciona el problema de lograr que los acreedores privados participen en las operaciones de rescate. La presión no oficial ejercida sobre los bancos acreedo- 
res por las autoridades reguladoras de sus países ha sido importante para que los bancos extranjeros y los países deudores alcancen un acuerdo lo antes posible, lo cual se logra con más facilidad en el caso de países deudores grandes, que en el caso de los pequeños. Además, gran parte de los préstamos internacionales adoptan la forma de valores -títulos valores como los bonos- y no de préstamos bancarios, por lo que es difícil lograr que los tenedores de bonos aunen esfuerzos voluntariamente con el fin de aliviar la deuda.

\section{DE LAS EXPERIENCIAS ADQUIRIDAS Y LAS ACCIONES MÁS EFICACES}

Las graves recesiones económicas que se produjeron después de las respuestas normativas iniciales del Fondo en cada uno de los países en crisis en 1997-1998 destacaron el hecho de que ni una política fiscal restrictiva, ni la restricción prolongada del crédito son políticas adecuadas cuando una economía se derrumba bajo la presión de una crisis financiera. Una política monetaria muy restrictiva al inicio de una crisis de pagos puede tener algunos beneficios si impulsa la confianza de los inversionistas y refuerza el atractivo de los activos financieros nacionales. Sin embargo, tales medidas también pueden socavar la confianza si debilitan un sistema financiero frágil y dejan al sector no financiero sin capital circulante. Otra de las conclusiones de la experiencia es que no se debe sobrecargar a un país con una serie de obligaciones de reforma estructural en medio de una crisis financiera, incluso si las medidas son necesarias para el desarrollo a largo plazo.

Los sistemas financieros de los países en desarrollo son en su conjunto más frágiles que la media mundial. Por ello, para reducir las posibilidades de que se produzcan crisis, las regulaciones financieras de esas economías deben ser más estrictas que las normas mundiales. En particular, la aplicación de los principios fundamentales para una supervisión bancaria eficaz debería ser más rigurosa y las normas relativas a la suficiencia de capital social deberían ser más estrictas que las Normas Generales del Comité de Basilea de $\mathrm{Su}$ pervisión Bancaria; ejemplo, endureciendo las condiciones relativas al capital con el fin de desalentar a los bancos domésticos a aceptar fondos nuevos.

A pesar de las diversas iniciativas que se han puesto en marcha ${ }^{\left({ }^{(8)}\right.}$, el carácter cada vez más mundializado de los mercados financieros y los vínculos crecientes entre las distintas categorías de operaciones financieras, ha dado lugar a propuestas para crear un organismo mundial para la regulación y supervisión financiera. Incluso con una mejor regulación prudencial, siempre habrá límites a los sistemas reguladores oficiales, en parte debido a la constante innovación de los mercados financieros. Por lo que las regulaciones deberían seguir haciendo hincapié en el fortalecimiento de los controles internos y la gestión del riesgo por las propias instituciones financieras.

En cuanto a las medidas preventivas, una de las opciones posibles del régimen de tipo de cambio, la oposición al establecimiento de controles en forma de desincentivos políticos a los movimientos de capital de corto plazo ha disminuido desde la crisis asiática. Además, los controles sobre el capital, las reservas de divisas representan la primera defensa de que disponen los gobiernos contra perturbaciones que pueden derivarse de la reversión de las corrientes de capital. Se ha sugerido que la cuantía de las reservas debe ser por lo menos igual a la deuda de un año. Otros factores que se han propuesto para determinar el monto adecuado de las reser- 
vas comprenden el déficit en cuenta corriente, el régimen del tipo de cambio, la variabilidad de la balanza de pagos y la incertidumbre que acompaña a la cuantificación de la deuda a corto plazo y las reservas. También se ha sugerido la necesidad de competir con el sector privado, la carga que representa el mantenimiento de reservas exigiendo a los bancos un mayor nivel de liquidez en moneda extranjera.

Como no es seguro, en caso de crisis, disponer de recursos suficientes para hacer frente a una situación de pánico general que afectara a varios países de manera más o menos simultánea, para afrontar una crisis de este tipo podría ser necesario que un prestamista de última instancia facilitara temporal y rápidamente grandes cantidades de liquidez a los países afectados. Los bancos centrales de los países desarrollados podrían hacer préstamos en su moneda a los bancos centrales de los países afectados, contra títulos de garantía en la moneda del prestatario -generalmente bonos del Estado. Otro mecanismo alternativo podría ser la creación temporal por el Fondo de un volumen considerable de Derechos Especiales de Giro (DEG), que los países podrían canjear mediante garantía a los bancos centrales de países con moneda clave, por sus monedas.

\section{REFORMA DE LA ARQUITECTURA FINANCIERA INTERNACIONAL}

El desplazamiento del centro de interés hacia la aplicación, evaluación y el perfeccionamiento de las políticas, significa que la asistencia técnica centrada en las necesidades particulares de cada país se ha convertido en un requisito clave para la eficacia de la labor de reforma de la arquitectura financiera internacional. La asistencia técnica y el apoyo resul- tan decisivos para asegurar que ningún país quede al margen de los esfuerzos encaminados a aumentar la capacidad de recuperación financiera en todo el mundo. Sin embargo, los episodios de crisis registrados en Argentina y en Turquía constituyen una clara advertencia de que aún queda mucho por hacer en materia de reforma de la arquitectura financiera internacional. Por otra parte, los responsables de la adopción de políticas tienen que estar dispuestos a hacer frente a posibles perturbaciones del sistema financiero y de la economía real, causadas por desequilibrios creados durante la última y larga fase de expansión económica.

Una parte fundamental de la estrategia para la reforma consiste en alentar a los países a que fortalezcan sus sistemas de políticas financieras y macroeconómicas mediante el establecimiento de normas y códigos de prácticas internacionales -éstas abarcan esferas tales como la transparencia en la gestión pública, la supervisión del sector bancario, la gestión empresarial y los marcos jurídicos e institucionales adecuados.

Inicialmente, la labor relativa a las normas y códigos internacionales ${ }^{(9)}$ se consideró como una manera de clasificar a los países en grupos distintos en función de su grado de cumplimiento de determinados reglamentos y normas uniformes, y los rezagados eran "castigados" por la comunidad internacional o los mercados financieros.

Actualmente, la opinión predominante es que el interés especial de las normas y códigos reside en un esfuerzo voluntario de cooperación encaminado a fortalecer el sistema mundial en su conjunto, y por tanto, el entorno económico y financiero internacional y nacional.

Para alcanzar mejor esos objetivos, el grado previsto de observancia de las normas y 
códigos internacionales por cada país debería tener en cuenta las distintas etapas del desarrollo económico y de los mercados de capitales, la capacidad administrativa y las políticas de reforma, así como las tradiciones culturales y jurídicas. En consecuencia, la observancia exige el establecimiento de prioridades, incluso de las normas básicas, con arreglo a las circunstancias específicas de cada país. Por otra parte, se deben encontrar medios para combinar una diferenciación adecuada en la aplicación y evaluación de las normas y la necesidad de mantener su universalidad.

La supervisión multilateral de las políticas económicas y financieras nacionales es el segundo pilar en orden de importancia de la arquitectura financiera internacional, después de las normas y códigos. La supervisión es tanto un servicio prestado a los distintos países como un bien público mundial, es el principal instrumento del que dispone la comunidad internacional para la prevención de las crisis; el FMI tiene la responsabilidad principal en materia de supervisión multilateral. Junto con esta institución mundial, existen acuerdos entre agrupaciones regionales y subregionales de países para ejercer una supervisión mutua.

Además de las políticas macroeconómicas tradicionales, la supervisión se centra en el aumento de la transparencia de las políticas de los países y en la elaboración y aplicación de las diversas normas mencionadas. También se está prestando una mayor atención a la determinación y a la reducción de las vulnerabilidades específicas, tanto nacionales como internacionales, que puedan aumentar la posibilidad de crisis financieras. En esta última esfera se ha prestado una especial atención a los mercados financieros, tanto nacionales como internacionales, $\mathrm{y}$ a sus consecuencias en la sostenibilidad de las corrientes de capitales. En ese sentido, hubo iniciativas para incrementar la comprensión, la vigilancia y la supervisión de los mercados mundiales de capitales y a fortalecer la capacidad para hacer frente a las crisis sistémicas relacionadas con el desarrollo de los mercados de capitales. Para este fin, el Fondo creó un Departamento de Mercados Internacionales de Capital, con la finalidad de complementar el fortalecimiento de los mercados financieros nacionales en los países importadores de capital, tratando de disminuir la volatilidad en la fuente de los fondos.

En abril de 2001, el Comité de Basilea publicó los resultados de un estudio de dos años de duración sobre las prácticas de divulgación pública de los bancos que realizan actividades en el plano internacional. La conclusión principal de este informe es que, aunque se han realizado importantes progresos durante los últimos años para mejorar la calidad y el nivel de la divulgación, las prácticas de divulgación han quedado atrás con respecto a los cambios en la manera en que los bancos llevan a cabo sus actividades y calculan sus riesgos.

En general, se han realizado esfuerzos para mejorar la gestión del riesgo en las instituciones financieras. A este respecto, se ha prestado especial atención a un nuevo Acuerdo de Basilea (Basilea II) sobre coeficientes mínimos de capital para sustituir el Acuerdo sobre coeficientes mínimos de capital de 1988. El principal objetivo del nuevo marco es armonizar mejor los coeficientes reglamentarios de los bancos que tienen riesgos latentes y alentar una supervisión más eficaz de parte de las autoridades encargadas de la superintendencia de los bancos.

En conclusión, para lograr un sistema financiero internacional más estable y propi- 


\section{Cuadro 1}

\begin{tabular}{|c|c|c|}
\hline \multicolumn{3}{|c|}{$\begin{array}{l}\text { NORMAS PARA EL FOMENTO DE SISTEMAS FINANCIEROS } \\
\text { NACIONALES SÓLIDOS }\end{array}$} \\
\hline & Normas y Códigos fundamentales & Entidad \\
\hline Difusión de datos & $\begin{array}{l}\text { Norma especial de difusión de datos/ } \\
\text { Sistema general de divulgación de datos }\end{array}$ & FMI \\
\hline $\begin{array}{l}\text { Transparencia fiscal } \\
\text { de la política }\end{array}$ & $\begin{array}{l}\text { Código de buenas prácticas de } \\
\text { transparencia fiscal }\end{array}$ & FMI \\
\hline $\begin{array}{l}\text { Transparencia en las } \\
\text { polít. monet. y financ. }\end{array}$ & $\begin{array}{l}\text { Código de buenas prácticas de } \\
\text { transparencia en las políticas monetarias } \\
\text { y financieras }\end{array}$ & FMI \\
\hline Supervisión bancaria & $\begin{array}{l}\text { Principios fundamentales para una } \\
\text { supervisión bancaria eficaz }\end{array}$ & BCBS \\
\hline Inspección de seguros & $\begin{array}{l}\text { Principios fundamentales en materia } \\
\text { de seguros }\end{array}$ & IAIS \\
\hline Reglam. de los valores & $\begin{array}{l}\text { Objetivos y principios de la } \\
\text { reglamentación en materia de valores }\end{array}$ & IOSCO \\
\hline Sistemas de pago & $\begin{array}{l}\text { Principios fundamentales para los } \\
\text { sistemas de pago sistémicamente import. }\end{array}$ & CPSS \\
\hline $\begin{array}{l}\text { Gobierno de las } \\
\text { sociedades }\end{array}$ & $\begin{array}{l}\text { Principios para el gobierno de las } \\
\text { sociedades }\end{array}$ & OCDE \\
\hline Contabilidad & Normas internacionales de Contabilidad & IASB \\
\hline Auditoría & Normas internacionales de Auditoría & IFAC \\
\hline $\begin{array}{l}\text { Regímenes de insolven. } \\
\text { y derechos de acreedor }\end{array}$ & $\begin{array}{l}\text { Principios y regímenes sobre insolvencia } \\
\text { efectiva y derechos de los acreedores }\end{array}$ & $\begin{array}{l}\text { Banco } \\
\text { Mundial }\end{array}$ \\
\hline $\begin{aligned} & \text { Fuente. } \text { FMI, Public Info } \\
& \text { BCBS Comité de } \\
& \text { IAIS Asociación I } \\
& \text { IOSCO Organize } \\
& \text { CPSS Comité de } \\
& \text { IASB Junta de N } \\
& \text { IFAC Federación }\end{aligned}$ & $\begin{array}{l}\text { n Notice N.o 17, marzo de } 2001 . \\
\text { visión Bancaria de Basilea } \\
\text { cional de Inspectores de Seguros } \\
\text { nternacional de Comisiones de Valores } \\
\text { as de Pago y Liquidaciones } \\
\text { Internacionales de Contabilidad } \\
\text { acional de Contadores }\end{array}$ & \\
\hline
\end{tabular}


cio al desarrollo, la responsabilidad incumbe en primer lugar a los responsables de formular las políticas en el plano nacional. Como las circunstancias económicas de los principales países desarrollados revisten una importancia decisiva en el establecimiento de un entorno económico internacional propicio para el desarrollo, éstos deben prestar más atención a la armonización de sus políticas con los objetivos y prioridades del desarrollo mundial. Por el lado del sector privado, que representa una proporción importante de las corrientes financieras internacionales, tienen obligaciones decisivas e indispensables en la prevención y solución de las crisis. A este respecto, se podría fomentar la intensificación del diálogo y un contacto activo y regular de doble vía sobre cuestiones de política, destinado a lograr una actividad más estable del sector privado, especialmente en los mercados emergentes.

\section{REFERENCIAS}

Duisenberg W. F., Acontecimientos y Tendencias Recientes en los Mercados Financieros Mundiales, Banco de México, 2001.

ECLAC, The Internacional Financial Crisis: An Eclac Perspective, Santiago, octubre 1998.

G-24, Grupo Intergubernamental de los 24 sobre Cuestiones Monetarias Internacionales y Desarrollo, Comunicado, abril 2001.

INVERTIA, La globalización de los Mercados Financieros, Centro Bursátil Monterrey, setiembre 2000.

, ¿Cómo Invertir en las Bolsas de valores?, Centro Bursátil Monterrey, abril 2001.
Naciones Unidas, Hacia una Nueva Arquitectura Financiera Internacional, Informe del Grupo de Trabajo del Comité Ejecutivo de Asuntos Económicos y Sociales, enero 1999.

, La Crisis Financiera y su Efecto en el Crecimiento y el Desarrollo, Especialmente en los Países en Desarrollo, Informe del Secretario General, octubre 1999.

Hacia la creación de un Sistema Financiero Internacional Estable que responda a los desafíos del desarrollo; especialmente en los Países en Desarrollo, Asamblea General, julio 2000.

, Hacia la creación de un Sistema Financiero Internacional Estable que responda a los desafíos del desarrollo; especialmente en los Países en Desarrollo, Asamblea General, Adición 1, julio 2000, y Adición 2, agosto 2000 .

Hacia una Arquitectura Internacional Fortalecida y estable que responda a las prioridades del crecimiento y desarrollo, especialmente en los Países en Desarrollo, y a la promoción de la equidad económica y social, Resolución aprobada por la Asamblea General, enero 2001.

, La Arquitectura Financiera Internacional y el Desarrollo, incluidas las transferencias netas de recursos entre los Países en Desarrollo y los Países Desarrollados, Asamblea General, julio 2001.

, La Arquitectura Financiera Internacional y el Desarrollo, incluidas las transferencias netas de recursos entre 
Países en Desarrollo y Países

Desarrollados, Asamblea Genera, Adición, julio 2001.

El Sistema Financiero Internacional y el Desarrollo, Informe del Secretario General, julio 2002.
United Nations, Towards a New Internacional Financial Architecture, Report of the Task Force of the Executive Comite on Economic and Social Affairs, January 1999.

, World Economic and Social Survey, 1999, Nueva York, 2000.

\section{NOTAS}

(1) Véase, Naciones Unidas, enero 1999. "La crisis de la deuda externa y las financieras internas tienen a su vez un altísimo costo social. Un alto porcentaje del costo del ajuste ante las crisis de endeudamiento recae en los actores pobres de la sociedad, que sólo reciben beneficios marginales en los períodos de auge firanciero", p. 28.

(2)Para enfrentar las crisis actuales se está exigiendo para que el FMI cree fondos de contingencia para asistir a los países afectados o contagiados, y también, para los que puedan convertirse en el futuro en víctimas de las crisis financieras mundiales. (Op. cit., p. 46).

(3) Se trata de los siete principales países industrializados, es un mecanismo que opera desde la década de los años setenta, que procura coordinar las políticas macroeconómicas de sus miembros. (Véase, Documento de Naciones Unidas. octubre 1999).

(4)La red de seguridad de los bancos tiene por objetivo básico evitar o controlar un pánico bancario general que podría obligar al cierre de los bancos fuertes como de los débiles, contraer la oferta monetaria y de crédito y causar una recesión económica. (Véase, Tendencias y Políticas en la Economía Mundial, en World Economic and Social Survey, 1999 pp.139-143.

(5)El sistema bancario en su conjunto actúa por lo general con un alto nivel de endeudamiento relativo al capital. Como los depósitos en un banco mantienen el volumen del saldo de sus depósitos, el banco puede utilizar una proporción importante de los fondos depositados para otorgar préstamos (o adquirir valores), reteniendo solo una porción pequeña de los depósitos en forma de activo líquido para atender la demanda diaria de efectivo. Por lo que el total de préstamos y de los valores que los bancos poseen es un múltiplo importante de las reservas en efectivo. (Op. cit., p. 139).

(6) El Grupo de los Diez quería reducir el riesgo de que una crisis bancaria en cualquiera de los países miembros pudiera extenderse más allá de las fronteras nacionales, así como evitar que las regulaciones menos estrictas de un país pudieran poner a los bancos de ese país en ventaja competitiva en los mercados mundiales. (Véase, Documento de Naciones Unidas, octubre 1999, pp. 9-10).

(7)En 1999 el FMI introdujo la línea de crédito contingente para países que el Fondo consideró que tenían políticas económicas sólidas. En caso de crisis, un país puede solicitar la aprobación del Directorio para activar la línea de crédito. La autorización debe concederse con rapidez y el Directorio decide sobre la cantidad que ha de liberar inmediatamente y en las fases siguientes. (Op. cit., p. 11).

(8) Además de operar en los programas introducidos en los países en crisis, el Fondo y el Banco Mundial establecieron en 1998 el Comité de Enlace para el Sector Financiero, con el fin de reforzar su colaboración en el fortalecimiento de sistemas financieros. Luego de la evaluación del Servicio Reforzado de Ajuste Estructural del Fondo en 1998, ambas instituciones se comprometieron a intensificar su colaboración para prestar asistencia a los países de bajos ingresos en que se centran los programas del Servicio Reforzado. A pesar de los esfuerzos realizados por la comunidad internacional, la reforma de la arquitectura financiera internacional está inconclusa a pesar de su urgencia. (Op. cit., p. 15).

(9)Las normas y códigos son elaborados en organismos especializados, así el FMI elaboró en materia de estadísticas financieras y transparencia de las políticas monetarias y financieras; el Comité de Supervisión Bancaria de Basilea elaboró lo relacionado a la cooperación bancaria, y en la OCDE se elaboró sobre gestión empresarial. (Véase, Documento de Naciones Unidas, julio 2001). 